\title{
Melatonin induces apoptosis in cholangiocarcinoma cell lines by activating the reactive oxygen species-mediated mitochondrial pathway
}

\author{
UMAWADEE LAOTHONG ${ }^{1-3,5}$, YUSUKE HIRAKU ${ }^{2}$, SHINJI OIKAWA ${ }^{2}$, KITTI INTUYOD ${ }^{3,4}$, \\ MARIKO MURATA $^{2 *}$ and SOMCHAI PINLAOR ${ }^{1,3^{*}}$ \\ ${ }^{1}$ Department of Parasitology, Faculty of Medicine, Khon Kaen University, Khon Kaen 40002, Thailand; \\ ${ }^{2}$ Department of Environmental and Molecular Medicine, Mie University Graduate School of Medicine, \\ Mie 514-8507, Japan; ${ }^{3}$ Liver Fluke and Cholangiocarcinoma Research Center, Faculty of Medicine, \\ Khon Kaen University, Khon Kaen 40002, Thailand; ${ }^{4}$ Biomedical Science Program, \\ Graduate School, Khon Kaen University, Khon Kaen 40002, Thailand
}

Received November 26, 2014; Accepted January 2, 2015

DOI: $10.3892 /$ or.2015.3738

\begin{abstract}
We previously demonstrated that melatonin could be used as a chemopreventive agent for inhibiting cholangiocarcinoma (CCA) development in a hamster model. However, the cytotoxic activity of melatonin in cancer remains unclear. In the present study, we investigated the effect of melatonin on CCA cell lines. Human CCA cell lines (KKU-M055 and KKU-M214) were treated with melatonin at concentrations of $0.5,1$ and $2 \mathrm{mM}$ for $48 \mathrm{~h}$. Melatonin treatment exerted a cytotoxic effect on CCA cells by inhibiting CCA cell viability in a concentration-dependent manner. Treatment with melatonin, especially at $2 \mathrm{mM}$, increased intracellular reactive oxygen species (ROS) production and in turn led to increased oxidative DNA damage and 8-oxodG formation. Moreover, melatonin treatment enhanced the production of cytochrome $c$ leading to apoptosis in a concentration-dependent manner, as indicated by increased expression of apoptosis-related proteins caspase- 3 and caspase- 7 . In conclusion, melatonin acts as a
\end{abstract}

Correspondence to: Dr Somchai Pinlaor, Department of Parasitology, Faculty of Medicine, Khon Kaen University, Khon Kaen 40002, Thailand

E-mail: psomec@kku.ac.th

Professor Mariko Murata, Department of Environmental and Molecular Medicine, Mie University Graduate School of Medicine, Tsu, Mie 514-8507, Japan

E-mail:mmurata@doc.medic.mie-u.ac.jp

Present address: ${ }^{5}$ Department of Community Health, Faculty of Public Health, Mahidol University, Bangkok 10400, Thailand

*Contributed equally

Key words: melatonin, apoptosis, cholangiocarcinoma, reactive oxygen species, DNA damage, mitochondrial pathway pro-oxidant by activating ROS-dependent DNA damage and thus leading to the apoptosis of CCA cells.

\section{Introduction}

Cholangiocarcinoma (CCA) is a devastating biliary cancer that poses continuing diagnostic and therapeutic challenges (1). There are several risk factors for CCA: mainly liver fluke infection, primary sclerosing cholangitis, biliary-duct cysts and hepatolithiasis (2). The highest prevalence of liver fluke Opisthorchis viverrini infection has been reported in Northeast Thailand, where CCA incidence is also high (3). CCA patients usually present after the disease is advanced and have a short survival outcome. Chemotherapy has not been shown to obviously improve survival in patients with CCA. Therefore, new strategies are needed to improve the treatment of this cancer.

Apoptosis, a form of programmed cell death, plays an important role in the homeostasis, development and prevention of cancer (4). Induction of apoptosis is considered a major goal of anticancer therapies $(5,6)$. Several chemopreventive agents exert their oncostatic effects via the production of reactive oxygen species (ROS), which ultimately disrupt the redox tone leading to cytostasis and/or cell death partly via the mitochondrial pathway (7). Mitochondria are believed to be the major source of ROS production (8). Furthermore, the mitochondrial pathway of apoptosis is triggered by various death signals, such as ROS and DNA damage. These signals promote binding of the pro-apoptotic protein Bax with the outer mitochondrial membrane, which disrupts the mitochondrial membrane potential, resulting in the release of apoptogenic factors such as cytochrome $c$ from the mitochondria to the cytosol. This in turns leads to activation of the caspase cascades and cell death $(9,10)$. Thus, enhancement of mitochondrial apoptosis may be a strategy for the treatment of cancer (11).

Melatonin, the major secretory product of the pineal gland, may exert differential effects for protection against cancer under certain conditions (12) with antioxidant property or oncostatic action (13). The oncostatic effects of melatonin 
involve the inhibition of neoplastic cell proliferation, intensified apoptosis, and decreased capacity to form metastases (14). Our previous study demonstrated the chemopreventive effect of melatonin on CCA hamsters (15). However, the cytotoxic effect of melatonin on CCA treatment remains unclear.

The present study aimed to investigate the cytotoxic effect of melatonin on human CCA cell lines. Human CCA cell lines with poor differentiation (KKU-M055) and well differentiation (KKU-M214) were treated with melatonin at concentrations of $0.5,1$, and $2 \mathrm{mM}$ for $48 \mathrm{~h}$. The viability of cells, generation of ROS, DNA damage formation, and expression of apoptotic-related proteins were assayed. The study revealed that melatonin exhibited a cytotoxic effect on CCA cell lines which points to a potential therapeutic role for melatonin in patients with advanced stage CCA.

\section{Materials and methods}

Cell culture and treatment. Human CCA cell lines KKU-M055 (poorly differentiated CCA) and KKU-M214 (well-differentiated CCA) were used in the present study. CCA cell lines were established and characterized from CCA patients hospitalized at the Faculty of Medicine, Khon Kaen University (16). Cells were cultured in Ham's F-12 medium supplemented with $10 \%$ fetal bovine serum, $100 \mathrm{U} / \mathrm{ml}$ penicillin and $100 \mu \mathrm{g} / \mathrm{ml}$ streptomycin at $37^{\circ} \mathrm{C}$ in a $5 \% \mathrm{CO}_{2}$ humidified atmosphere. Melatonin (Huanggang Innovation Biochemicals, Hubei, China) was dissolved in dimethyl sulfoxide (DMSO) before being diluted with medium with a final DMSO concentration of $0.1 \%$. Cells were treated with melatonin at concentrations of $0.5,1$ and $2 \mathrm{mM}$, or vehicle (0.1\% DMSO) for $48 \mathrm{~h}$.

Cell viability assay. Cell viability was assayed by 3-(4,5-dimethylthiazol-2-yl)-2,5-diphenyl tetrazolium bromide (MTT; Invitrogen Life Technologies, Carlsbad, CA, USA). Briefly, cells were plated at a density of $1 \times 10^{4}$ cells/ well into 96-well plates. After overnight growth, the cells were treated with $1 \mathrm{nM}, 1 \mu \mathrm{M}, 0.5,1$ and $2 \mathrm{mM}$ of melatonin for 24, 48, and $72 \mathrm{~h}$. After treatment, the cells were incubated for $4 \mathrm{~h}$ with $5 \mathrm{mg} / \mathrm{ml}$ of MTT reagent and lysed with DMSO. The absorbance was monitored with a microplate reader at a wavelength of $570 \mathrm{~nm}$ (reference wavelength at $655 \mathrm{~nm}$ ).

Measurement of intracellular ROS. Evaluation of intracellular oxidants was carried out using the fluorescent probe 5-(and -6)-chloromethyl-2',7'-dichlorodihydrofluorescein diacetate, acetyl ester (CM- ${ }_{2}$ DCFDA; Invitrogen Life Technologies). Briefly, after treatment with melatonin for $48 \mathrm{~h}$, the cells were incubated with PBS containing $\mathrm{CM}-\mathrm{H}_{2} \mathrm{DCFDA}$ at a final concentration of $5 \mu \mathrm{M}$ at $37^{\circ} \mathrm{C}$ for $30 \mathrm{~min}$. The cells were then harvested by trypsinization and washed twice with PBS. The cells were suspended in PBS and analyzed on a flow cytometer (FACScan; Becton-Dickinson, San Jose, CA, USA).

Immunostaining of 8-oxo-7, 8-dihydro-2'-deoxyguanosine (8-oxodG). To evaluate the simultaneous localization of 8-oxodG in nuclear and mitochondrial DNA, the cells were labeled with $100 \mathrm{nM}$ MitoTracker ${ }^{\circledR}$ Red CMXRos probe (mitochondria-specific) (Invitrogen Life Technologies) for $15 \mathrm{~min}$ at $37^{\circ} \mathrm{C}$. After staining, the cells were washed in PBS
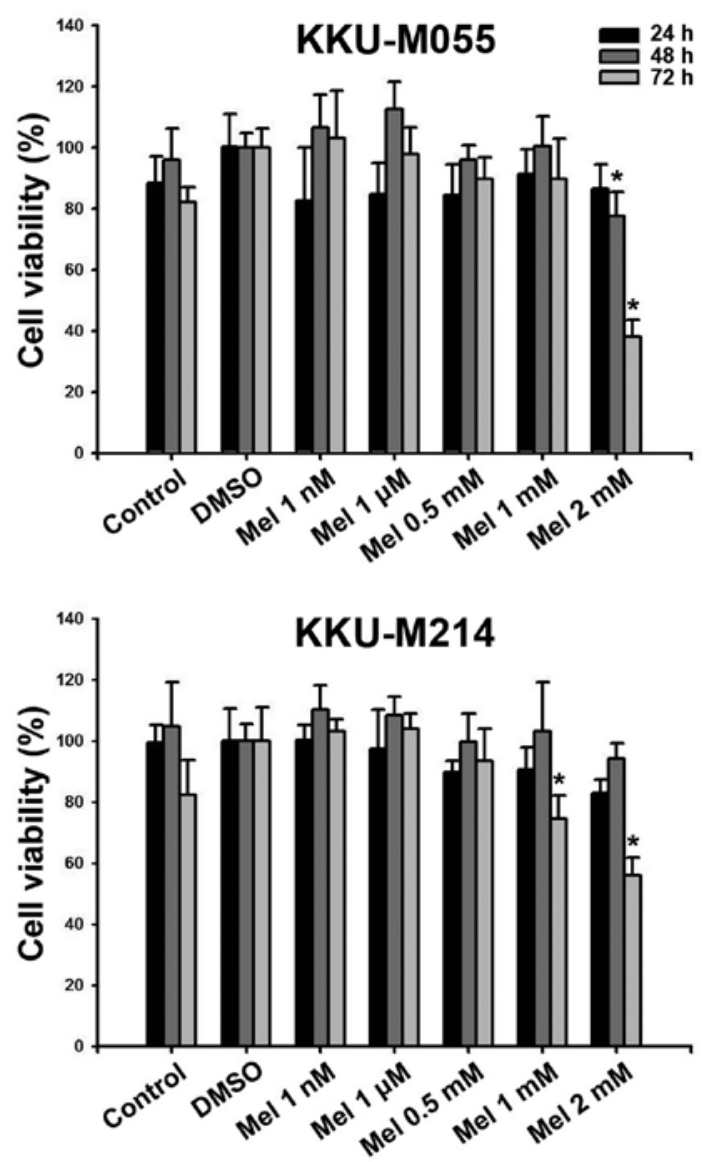

Figure 1. Effect of melatonin treatment on cell viability as measured by MTT assay. KKU-M055 and KKU-M214 cells were treated with melatonin (1 nM, $1 \mu \mathrm{M}$ and $0.5,1$, and $2 \mathrm{mM}$ ) for 24,48 , and $72 \mathrm{~h}$. Results are presented as mean $\pm \mathrm{SD}$. ${ }^{*} \mathrm{P}<0.05$ as compared to the DMSO group. DMSO represents vehicle control with $0.1 \%$ DMSO. DMSO, dimethyl sulfoxide.

and fixed with $4 \%$ formaldehyde in PBS for $10 \mathrm{~min}$. The cells were then permeabilized with $0.5 \%$ Triton X-100 in PBS for 3 min and washed with PBS before being blocked with 5\% skim milk for $60 \mathrm{~min}$. Subsequently, the cells were incubated with mouse monoclonal anti-8-oxodG antibody (Japan Institute for the Control of Aging, Fukuroi, Japan) at 1:200 dilution overnight at room temperature and then incubated with Alexa 488-labeled goat antibody against mouse $\operatorname{IgG}$ at 1:400 dilution for $3 \mathrm{~h}$ at room temperature. The cells were mounted and visualized under a fluorescence microscope (Axiovert 200; Carl Zeiss, Göttingen, Germany). The excitation/emission wavelengths for MitoTracker ${ }^{\circledR}$ Red CMXRos were $579 / 599 \mathrm{~nm}$, respectively.

Measurement of cell death. Cell death was quantified using TACS $^{\text {TM }}$ Annexin V kits (Trevigen, Gaithersburg, MD, USA) as per the manufacturer's instructions. Briefly, the cells were collected by centrifugation at $300 \mathrm{x} \mathrm{g}$ for $10 \mathrm{~min}$ and washed with PBS. The cells were then resuspended in $100 \mu \mathrm{l}$ of Annexin V/PI incubation reagent for $15 \mathrm{~min}$ at room temperature, followed by addition of $400 \mu \mathrm{l}$ of $1 \mathrm{X}$ binding buffer and processing by flow cytometry. The following staining criterion was used for characterization: cells that were not stained by either Annexin V or PI were considered healthy, cells stained by only Annexin $\mathrm{V}$ were considered early apoptotic, and cells 
A

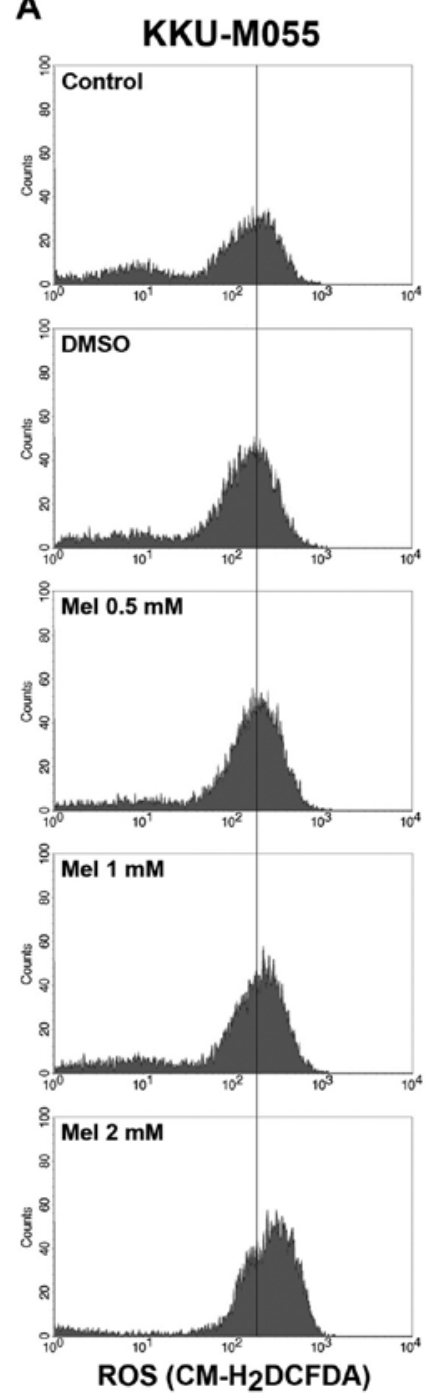

KKU-M214
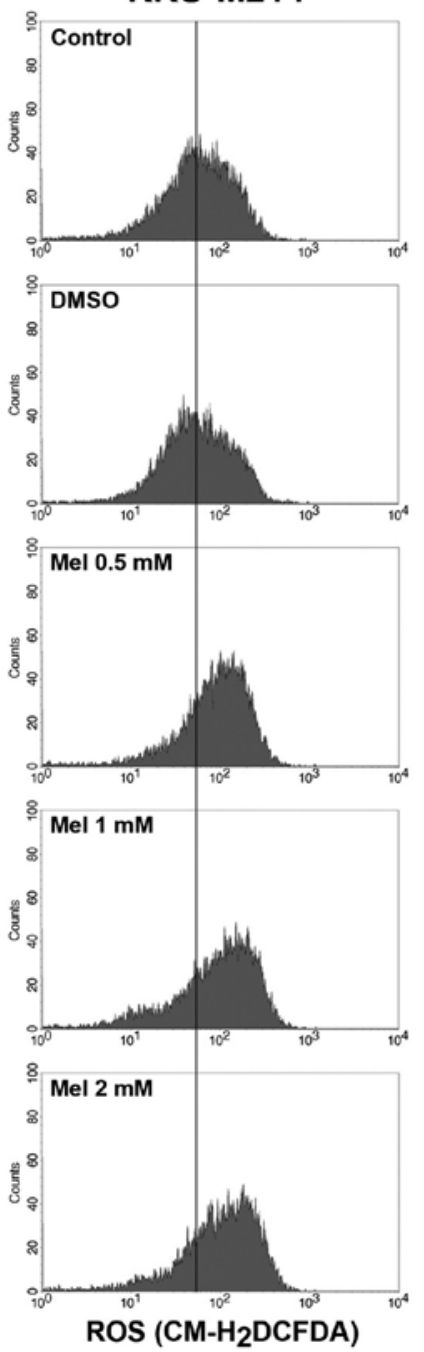

B
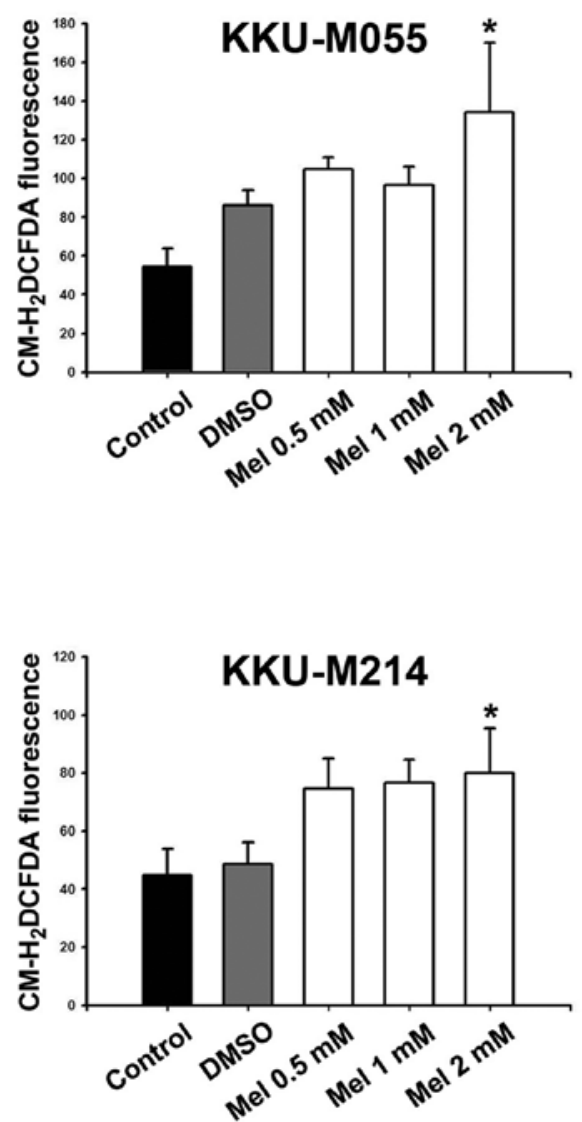

Figure 2. Effect of melatonin treatment on ROS production. ROS levels were measured using CM-H $\mathrm{H}_{2}$ DCFDA fluorescence by flow cytometry. (A) Histogram of CM- $\mathrm{H}_{2}$ DCFDA intensities in a representative experiment. Vertical lines indicate the mean fluorescence values for the vehicle control cells. (B) The geometric mean of fluorescence $\pm \mathrm{SD}$ analyzed using FACScan ${ }^{\mathrm{TM}}$ system. ${ }^{*} \mathrm{P}<0.05$ as compared to the DMSO group. DMSO represents vehicle control with $0.1 \%$ DMSO. ROS, reactive oxygen species; DMSO, dimethyl sulfoxide.

stained by both PI and Annexin V were considered late apoptotic or necrotic.

Western blot analysis. Protein from the whole cell lysates was extracted using RIPA buffer $(50 \mathrm{mM}$ Tris- $\mathrm{HCl}, 150 \mathrm{mM} \mathrm{NaCl}$, $1 \%$ Triton X-100, $0.5 \%$ sodium deoxycholate and $0.1 \%$ SDS). Protein was separated on 12-15\% SDS-PAGE, transferred onto a polyvinylidenedifluoride membrane (GE Healthcare Biosciences Corp., Piscataway, NJ, USA) and immunoblotted with specific antibodies. The primary antibodies used were rabbit polyclonal anti-Bcl2 (ab7973), rabbit polyclonal anti-caspase-3 (ab44976), rabbit polyclonal anti-inducible nitric oxide synthase (iNOS) (ab15323; Abcam, Cambridge, MA, USA), goat polyclonal anti-caspase-7 (sc-6138) and rabbit polyclonal anti-cytochrome $c$ (sc-7159; Santa Cruz Biotechnology, Santa Cruz, CA, USA). Rabbit polyclonal antiGAPDH antibody (ab77109; Abcam) was used as a sample loading control. Immunostained protein bands were detected with an enhanced chemiluminescence kit (GE Healthcare Biosciences Corp.).
Statistical analysis. The data are expressed as mean \pm SD. Statistical analysis was conducted using one-way analysis of variance (ANOVA) using SPSS version 11.5 (SPSS Inc., Chicago, IL, USA). A P $<0.05$ was considered to indicate a statistically significant result.

\section{Results}

Melatonin decreases cell viability. To investigate the effect of the concentration and the duration of melatonin treatment on the viability of cells, KKU-M055 and KKU-M214 cells were treated with different concentrations of melatonin $(1 \mathrm{nM}, 1 \mu \mathrm{M}$ and $0.5,1$ and $2 \mathrm{mM}$ ) for 24,48 , and $72 \mathrm{~h}$. The result of the MTT assay showed that melatonin decreased the percentage of viable cells in a concentration and time-dependent manner. Treatment with physiological $(1 \mathrm{nM})$ or low pharmacological $(1 \mu \mathrm{M})$ concentrations of melatonin did not demonstrate a significant effect. Treatment with high pharmacological concentrations of melatonin ( 1 and $2 \mathrm{mM})$ significantly decreased cell viability compared to the DMSO-treated group 

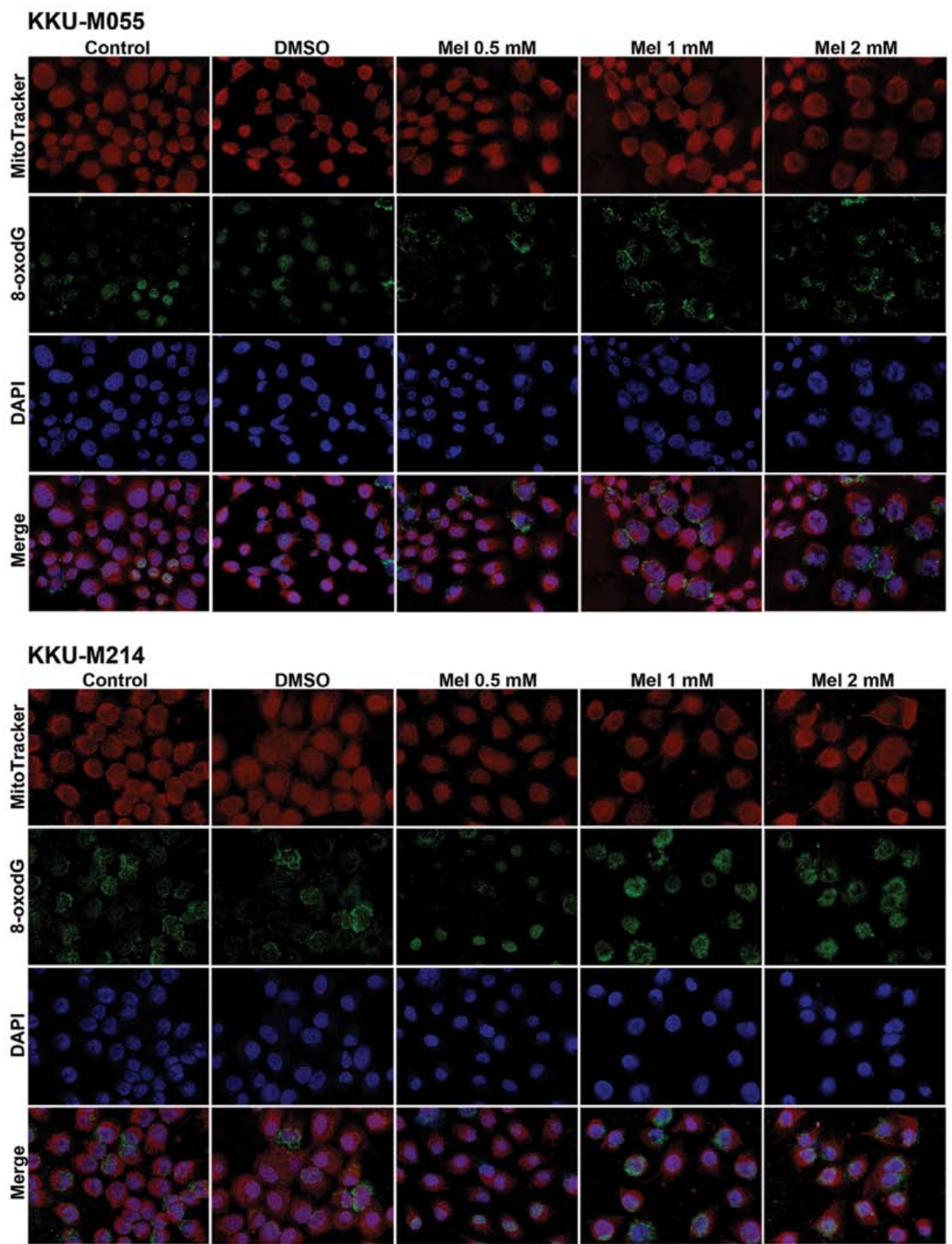

Figure 3. Fluorescence microscopy images of melatonin-treated cells for $48 \mathrm{~h}$. Mitochondria were visualized using MitoTracker (red). DNA damage was measured by 8-oxodG (green). Nuclei were stained with DAPI (blue). Overlay of the 3 channels shows colocalization of MitoTracker, 8-oxodG, and DAPI.

(Fig. 1). Therefore, concentrations of $0.5,1$ and $2 \mathrm{mM}$ and an incubation time of $48 \mathrm{~h}$ were used in the subsequent experiments.

Melatonin enhances ROS production. The ROS generation in KKU-M055 and KKU-M214 cells after treatment with different concentrations $(0.5,1$, and $2 \mathrm{mM})$ of melatonin for $48 \mathrm{~h}$ was measured by CM- $\mathrm{H}_{2}$ DCFDA fluorescence. The result showed that melatonin treatment increased ROS production in a concentration-dependent manner (Fig. 2). At a high concentration $(2 \mathrm{mM})$ of melatonin treatment, it significantly increased $\mathrm{CM}-\mathrm{H}_{2}$ DCFDA fluorescence when compared with the DMSO-treated group (Fig. 2B). We examined the protein expression of iNOS, an enzyme that generates free radical nitric oxide (NO) by western blot analysis. The result revealed that melatonin treatment increased iNOS expression in a concentration-dependent manner (Fig. 5).

Melatonin induces DNA damage. Since ROS can cause oxidative damage to DNA, we measured the damage of DNA caused by melatonin treatment by immunostaining of 8-oxodG for $48 \mathrm{~h}$ after melatonin treatment. The results showed that melatonin treatment coincided with an increase in 8-oxodG-positive cells in a concentration-dependent manner. 
A KKU-M055
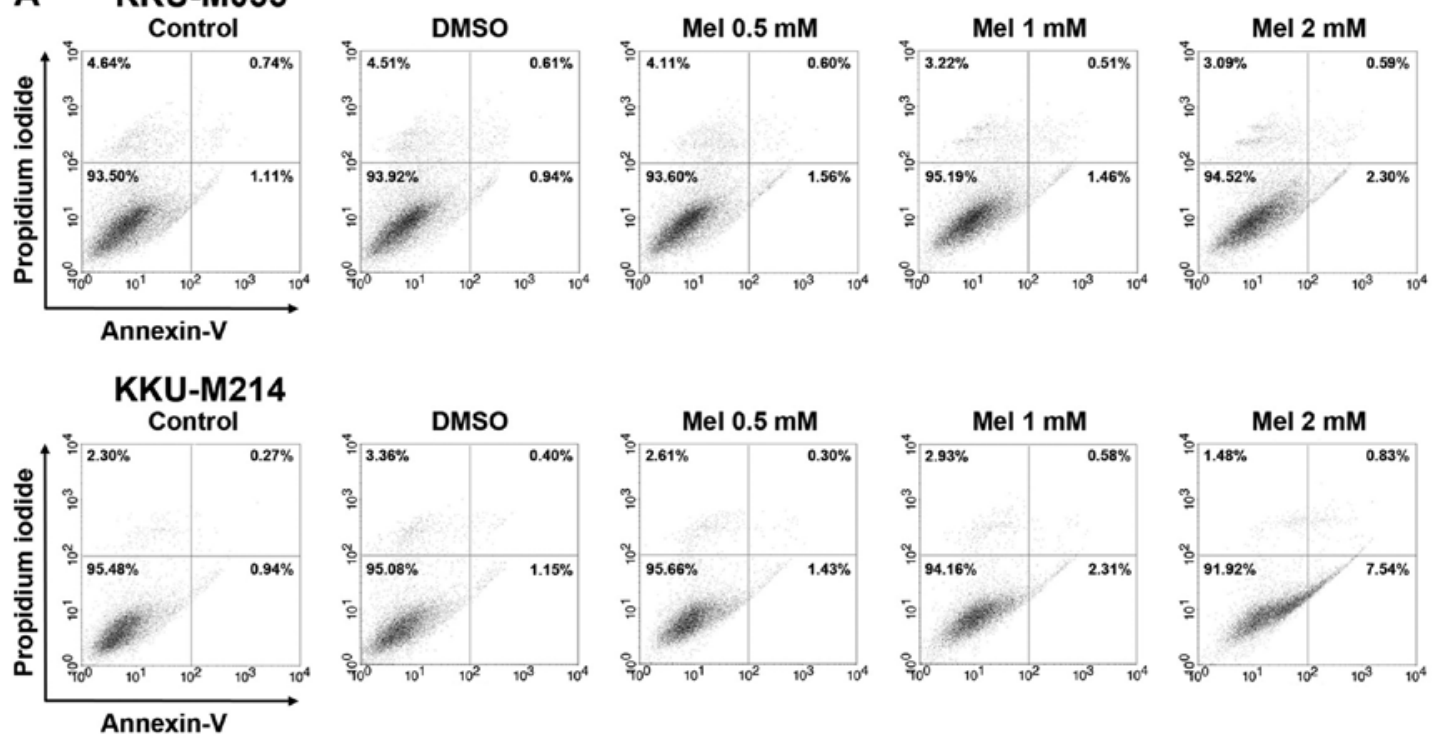

B
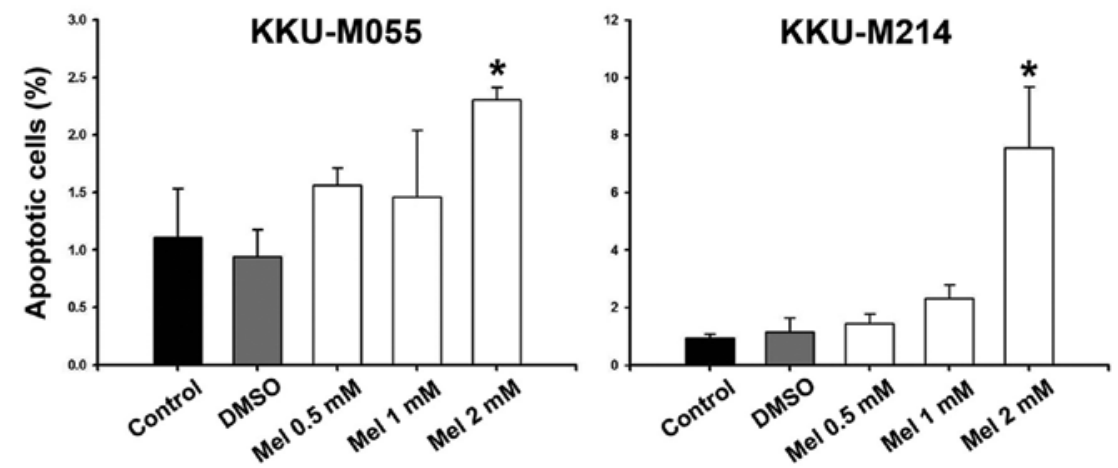

Figure 4. Effect of melatonin treatment on apoptosis in CCA cells. Apoptosis of CCA cells treated with $0.5,1$, and $2 \mathrm{mM}$ melatonin at $48 \mathrm{~h}$ was evaluated using Annexin V-FITC/PI staining and flow cytometric analysis. (A) Representative dot plots showing distribution of cells under different treatment conditions after flow cytometry. Fluorescence intensity for Annexin V/FITC is plotted on the x-axis, and PI is plotted on the y-axis. (B) The percentage of apoptotic cells (lower right quadrant) under different treatment conditions is shown. Results are presented as mean \pm SD of 3 separate experiments. ${ }^{*}<0.05$ as compared to the DMSO group. DMSO represents vehicle control with 0.1\% DMSO. DMSO, dimethyl sulfoxide; CCA, cholangiocarcinoma.

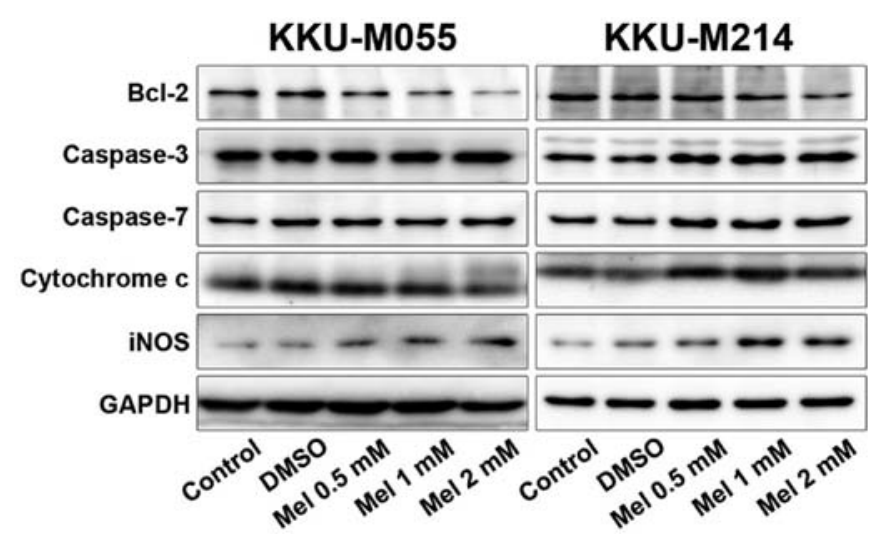

Figure 5. Effect of melatonin treatment on the expression of apoptosisrelated proteins. The expression levels of Bcl-2, caspase-3 and caspase-7, cytochrome $c$, and iNOS proteins of CCA cells treated with $0.5,1$, and $2 \mathrm{mM}$ melatonin for $48 \mathrm{~h}$ were examined by western blot analysis. GAPDH expression was used as an internal control. iNOS, inducible nitric oxide synthase; CCA, cholangiocarcinoma.

Notably, the 8-oxodG-positive area in the control and DMSOtreated groups was mostly found in the nucleus while this area in the melatonin-treated group was distributed to the cytoplasm and mitochondria, as shown by the result of merging the 8-oxodG and MitoTracker signals (Fig. 3).

Melatonin induces apoptosis. The apoptosis caused by different concentrations of melatonin was evaluated by flow cytometry of Annexin V/PI. The results showed that melatonin increased the percentage of apoptotic cells in a concentration-dependent manner. Compared to the DMSO-treated cells, there was a significant increase in the proportion of cells in early apoptosis (Annexin $\mathrm{V}^{+} / \mathrm{PI}^{-}$) after $2 \mathrm{mM}$ melatonin treatment (Fig. 4). Furthermore, we examined the expression of apoptosis-related proteins by western blot analysis. Melatonin treatment promoted the activation of caspase- 3 and caspase- 7 , and cytochrome $c$ while inhibiting the expression of the antiapoptotic protein $\mathrm{Bcl}-2$ (Fig. 5).

\section{Discussion}

Melatonin has been shown to have a wide range of physiological functions in humans and has been implicated in the disruption of neoplastic processes $(17,18)$. The physiological 
actions of melatonin are partly attributed to its antioxidant activity (19). Under certain conditions, melatonin may also exert oxidant effects, particularly in cancer cells (20). In the present study, we demonstrated that high pharmacological concentrations of melatonin exhibited a cytotoxic effect on human CCA cell lines. Our results showed that melatonin enhanced ROS-dependent DNA damage, leading to apoptosis and reducing the viability of cells. This suggests that melatonin induces tumor cell death by its pro-oxidant activity $(20,21)$.

Melatonin may act as either a pro-oxidant or an antioxidant in different contexts in order to exert an antitumor effect (12). It is well known as an antioxidant, free radical scavenger and oncostatic agent in preventing tumors at initiation, promotion and progression steps in a variety of cancer types such as breast, colorectal, hepatocellular, and bile duct cancer (22-25). In our previous study, we showed that melatonin exerted chemopreventive effects on $O$. viverrini infection (26) and on $O$. viverrini plus $N$-nitrosodimethylamine-induced CCA in hamsters (15) by reducing oxidative and nitrative DNA damage, liver injury, and tumor mitochondrial pathways of apoptosis. On the other hand, here, we found that melatonin exhibited pro-oxidant activity instead of an antioxidant effect in CCA cells, suggesting that melatonin may be a doubleedged sword acting in cancer under certain conditions (12).

Although there is much evidence that melatonin exerts antioxidant activity, a few studies using cultured cells have revealed that melatonin may generate ROS at pharmacologically active concentrations ( $\mu \mathrm{M}$ to $\mathrm{mM}$ range) and therefore may have pro-oxidant activity under some conditions (12). Our results showed that melatonin was able to stimulate production of intracellular ROS as evidenced by the increase in $\mathrm{CM}-\mathrm{H}_{2} \mathrm{DCFDA}$ fluorescence. Treatment of human CCA cell lines with melatonin at a concentration of $2 \mathrm{mM}$ increased ROS generation, but this effect was not observed at 0.5 or $1 \mathrm{mM}$ of melatonin. Stimulation of ROS production by $2 \mathrm{mM}$ melatonin in CCA cell lines was similar to the previous finding that highdose $(1 \mathrm{mM})$ melatonin caused stimulation of ROS production in human leukaemia cells, but did not after treatment with a melatonin dose lower than $10 \mu \mathrm{M}$ (27). Taken together, these results demonstrate that melatonin induces the production of ROS that may account for the cytotoxicity of melatonin at high concentrations $(20,21,27)$.

Mechanistically, the production of ROS by melatonin in high doses may be explained by its interaction with calmodulin, the mitochondrial complex III or the mitochondrial transition pore (12). The pro-oxidant, cytotoxic and pro-apoptotic effects of melatonin on human CCA cell lines were similar to the effects of melatonin treatment on human promyelocytic leukaemia HL-60 cells observed previously $(20,28)$. Thus, the pro-oxidant action of melatonin may assist in restraining CCA cell growth, which may be useful for treating patients with otherwise untreatable metastatic solid tumors (29).

Treatment with melatonin induced ROS production, which was concomitant with apoptosis and a loss of CCA cell viability. These results are consistent with previous observations that other cancer cells are sensitive to melatonin, particularly liver cancer cells $(30,31)$. Melatonin treatment also induced a decrease in the expression of the anti-apoptotic protein $\mathrm{Bcl}-2$ and an increase in the expression of cyto- chrome $c$ and apoptotic-associated caspases (caspase- 3 and caspase-7), all of which are indicators of apoptotic cell death. Moreover, 8-oxodG in melatonin-treated cells was localized to the cytoplasm and mitochondria as shown by the result of merging 8-oxodG and MitoTracker (Fig. 3), while in the control and DMSO-treated groups 8-oxodG was mostly found in the nucleus, indicating that melatonin induced mitochondrial DNA damage. Taken together, these data suggest that the increase in ROS production is responsible for the pro-apoptotic effect of high concentrations of melatonin in CCA cells.

We previously demonstrated that melatonin can attenuate apoptosis in hamster CCA. In contrast, we here found that treatment with melatonin at $2 \mathrm{mM}$ induced apoptosis in human CCA cells, but at lower concentrations of 0.5 and $1 \mathrm{mM}$ melatonin treatment had no effect on apoptosis. Previous studies have shown that melatonin treatment of non-small cell lung cancers (NSCLCs) after genotoxic stress induced by irradiation with UV increased apoptosis, but no apoptotic effect was observed in NSCLC cell lines without genotoxic damage (32). Furthermore, induction of apoptosis by melatonin was dosedependent in the high stress conditions, the predominance of DNA damage, in NSCLC cell lines. Taken together, we suggest that melatonin exerts effects on the apoptosis pathway according to stress conditions and the dose of melatonin. This phenomenon may be caused by increased oxidative stress of cancer cells as a result of their elevated metabolism. Thus, melatonin treatment may be useful in impairing the ROS buffering capacity of cancer cells (28). We did not test the cytotoxic effect of melatonin on normal cholangiocytes in the present study; however, previous studies have shown that melatonin does not confer any observable cytotoxic effects on hepatocytes in normal hamsters (26).

In conclusion, melatonin treatment in human CCA cell lines increased intracellular ROS, which was associated with significant cytotoxicity and activation of caspase activities. Melatonin may exert a pro-oxidant cytotoxic effect on human CCA cell lines and thereby reduce the viability of cancer cells by activating ROS-dependent DNA damage and inducing apoptosis. The activation of ROS production by melatonin is associated with cytotoxicity in CCA cells, but only at high concentrations of melatonin. We suggest that melatonin could be used as a supplement to classical anticancer drugs in therapies used to treat CCA patients. Confirming the efficacy and safety of melatonin in cancer treatment will require further study in CCA patients.

\section{Acknowledgements}

The present study was supported by The Thailand Research Fund through Royal Golden Jubilee Ph.D. Program (to U.L. and S.P.), the Thailand Research Fund (RMU5380016), and The Higher Education Research Promotion and National Research University Project of Thailand, Office of the Higher Education Commission, through the Health Cluster (SHeP-GMS), Khon Kaen University. This study was partly supported by Grants-in-Aids for Scientific Research from the Ministry of Education, Culture, Sports, Science and Technology of Japan (no. 25293149). We thank Dr Justin Reese, Research Affairs, Faculty of Medicine, Khon Kaen University, Thailand for critically reading and improving this manuscript. 


\section{References}

1. Anderson CD, Pinson CW, Berlin J and Chari RS: Diagnosis and treatment of cholangiocarcinoma. Oncologist 9: 43-57, 2004.

2. Tyson GL and El-Serag HB: Risk factors for cholangiocarcinoma. Hepatology 54: 173-184, 2011.

3. IARC: A Review of Human Carcinogens: Opisthorchis viverrini and Clonorchis sinensis. IARC Monogr Eval Carcinog Risks Hum 100B: 341-370, 2012.

4. Gerl R and Vaux DL: Apoptosis in the development and treatment of cancer. Carcinogenesis 26: 263-270, 2005.

5. Kaufmann SH and Earnshaw WC: Induction of apoptosis by cancer chemotherapy. Exp Cell Res 256: 42-49, 2000.

6. Hannun YA: Apoptosis and the dilemma of cancer chemotherapy. Blood 89: 1845-1853, 1997.

7. Hail N Jr and Lotan R: Cancer chemoprevention and mitochondria: targeting apoptosis in transformed cells via the disruption of mitochondrial bioenergetics/redox state. Mol Nutr Food Res 53: 49-67, 2009.

8. Turrens JF: Mitochondrial formation of reactive oxygen species. J Physiol 552: 335-344, 2003.

9. Green DR and Reed JC: Mitochondria and apoptosis. Science 281: 1309-1312, 1998.

10. Desagher S and Martinou JC: Mitochondria as the central control point of apoptosis. Trends Cell Biol 10: 369-377, 2000.

11. Kasibhatla $\mathrm{S}$ and Tseng B: Why target apoptosis in cancer treatment? Mol Cancer Ther 2: 573-580, 2003.

12. Zhang HM and Zhang Y: Melatonin: a well-documented antioxidant with conditional pro-oxidant actions. J Pineal Res 57: 131-146, 2014.

13. Bartsch $\mathrm{C}$, Bartsch $\mathrm{H}$ and Karasek M: Melatonin in clinical oncology. Neuro Endocrinol Lett 23: 30-38, 2002.

14. Jung B and Ahmad N: Melatonin in cancer management: progress and promise. Cancer Res 66: 9789-9793, 2006.

15. Laothong U, Pinlaor P, Boonsiri P, et al: Melatonin inhibits cholangiocarcinoma and reduces liver injury in Opisthorchis viverrini-infected and $N$-nitrosodimethylamine-treated hamsters. J Pineal Res 55: 257-266, 2013.

16. Sripa B, Leungwattanawanit S, Nitta T, et al: Establishment and characterization of an opisthorchiasis-associated cholangiocarcinoma cell line (KKU-100). World J Gastroenterol 11: 3392-3397, 2005.

17. Sanchez-Barcelo EJ, Cos S, Fernandez R and Mediavilla MD: Melatonin and mammary cancer: a short review. Endocr Relat Cancer 10: 153-159, 2003.

18. Hill SM, Frasch T, Xiang S, Yuan L, Duplessis T and Mao L: Molecular mechanisms of melatonin anticancer effects. Integr Cancer Ther 8: 337-346, 2009.
19. Barrenetxe J, Delagrange P and Martinez JA: Physiological and metabolic functions of melatonin. J Physiol Biochem 60: 61-72, 2004.

20. Bejarano I, Espino J, Barriga C, Reiter RJ, Pariente JA and Rodriguez AB: Pro-oxidant effect of melatonin in tumour leucocytes: relation with its cytotoxic and pro-apoptotic effects. Basic Clin Pharmacol Toxicol 108: 14-20, 2011.

21. Rodriguez C, Martin V, Herrera F, et al: Mechanisms involved in the pro-apoptotic effect of melatonin in cancer cells. Int J Mol Sci 14: 6597-6613, 2013

22. Carbajo-Pescador S, Garcia-Palomo A, Martin-Renedo J, Piva M, Gonzalez-Gallego J and Mauriz JL: Melatonin modulation of intracellular signaling pathways in hepatocarcinoma HepG2 cell line: role of the MT1 receptor. J Pineal Res 51: 463-471, 2011.

23. Han Y, Demorrow S, Invernizzi P, et al: Melatonin exerts by an autocrine loop antiproliferative effects in cholangiocarcinoma: its synthesis is reduced favoring cholangiocarcinoma growth. Am J Physiol Gastrointest Liver Physiol 301: G623-G633, 2011.

24. Motilva V, Garcia-Maurino S, Talero E and Illanes M: New paradigms in chronic intestinal inflammation and colon cancer: role of melatonin. J Pineal Res 51: 44-60, 2011.

25. Proietti S, Cucina A, Reiter RJ and Bizzarri M: Molecular mechanisms of melatonin's inhibitory actions on breast cancers. Cell Mol Life Sci 70: 2139-2157, 2013.

26. Laothong U, Pinlaor P, Hiraku Y, et al: Protective effect of melatonin against Opisthorchis viverrini-induced oxidative and nitrosative DNA damage and liver injury in hamsters. J Pineal Res 49: 271-282, 2010

27. Buyukavci M, Ozdemir O, Buck S, Stout M, Ravindranath Y and Savasan S: Melatonin cytotoxicity in human leukemia cells: relation with its pro-oxidant effect. Fundam Clin Pharmacol 20: 73-79, 2006.

28. Bejarano I, Espino J, Marchena AM, et al: Melatonin enhances hydrogen peroxide-induced apoptosis in human promyelocytic leukaemia HL-60 cells. Mol Cell Biochem 353: 167-176, 2011.

29. Lissoni P, Paolorossi F, Tancini G, et al: A phase II study of tamoxifen plus melatonin in metastatic solid tumour patients. $\mathrm{Br}$ J Cancer 74: 1466-1468, 1996.

30. Martin-Renedo J, Mauriz JL, Jorquera F, Ruiz-Andres O Gonzalez P and Gonzalez-Gallego J: Melatonin induces cell cycle arrest and apoptosis in hepatocarcinoma HepG2 cell line. J Pineal Res 45: 532-540, 2008.

31. Osseni RA, Rat P, Bogdan A, Warnet JM and Touitou Y: Evidence of prooxidant and antioxidant action of melatonin on human liver cell line HepG2. Life Sci 68: 387-399, 2000.

32. Kim W, Jeong JW and Kim JE: CCAR2 deficiency augments genotoxic stress-induced apoptosis in the presence of melatonin in non-small cell lung cancer cells. Tumour Biol 135: 10919-10929, 2014. 